\title{
CHEMOPREVENTIVE EFFECT OF CAPSAICIN IN EXPERIMENTALLY INDUCED HAMSTER BUCCAL POUCH CARCINOGENESIS (IMMUNOHISTOCHEMICAL STUDY BCL-2)
}

\author{
Mohamed Abdelrahman Mohamed* and Ali Abdullah AlQarni**
}

\begin{abstract}
Aim:To investigate immunohistochemically the effect of chemopreventive capsaicin in epithelial alteration (Epithelial dysplasia) \& carcinogenesis which induced in hamsters.

Methods: Hematoxylin and Eosin (H\&E) and Streptavidin-biotin immunoperoxidase staining techniques was used to detect the expression of Bcl-2 in three groups (G1, G2 and G3). Evaluation of immunostaining was done using computer image analyzer system \& statistical analysis.

Results: showed that there was a statistically highly significant difference $(\mathrm{P}<0.01)$ in means values of all groups $(\mathrm{G} 2>\mathrm{G} 1, \mathrm{G} 3)(\mathrm{G} 3>\mathrm{G} 1)$.

Conclusion:Capsaicin play an important role in reduction and prevention of transformation of epithelial dysplasia into malignant tumor especially oral squamous cell carcinoma. The liquid form of capsaicin may be considered as a new line of treatment in oral dysplasia or even in oral squamous cell carcinoma and the Bcl-2 expression evidenced this reduction of abnormality in epithelial dysplasia.
\end{abstract}

KEYWORDS: Oral Epithelial Dysplasia, chemopreventive effect, capsaicin, Bcl-2 , induced hamster carcinogenesis.

\section{INTRODUCTION}

Oral Epithelial Dysplasia is a lesion of the epithelium that represents avariation in the differentiation and maturation of cells with tendencygreater multiplication of cells and diminished differentiation. The steps taken to achieve a process of cancer developing OED may lapse, remain unvarying, or proceed to Squamous Cell Carcinoma (SCC) resting on a coalition of environmental, genetic, epigenetic and immune factors. Oral Epithelial Dysplasia is a distinguishable histological variation that is present in some Potentially Malignant Disorders (PMDs). The most common PMDs in connection to Epithelial Dysplasia (ED) are leukoplakia, erythroplakia, oral submucous fibrosis, and lichen planus.

\footnotetext{
* Assistant Professor of Oral Pathology , Faculty of Dentistry , Al-Azhar University.
}

** Teaching Assistant of Oral Medicine , Faculty of Dentistry, Taif University 
All presently obtainable systems classify Epithelial Dysplasia to at any rate three levels mainly based on the degree of participation of the epithelium by immature cells and the degree of superficial maturation. They are all highly discrete and as a result not very reproducible. In the nonappearance of agreement for the best system, the WHO system with the mild, moderate, severe and carcinoma in situ grades is the most commonly followed.

The oral cavity cancer accounts for over $2 \%$ of all malignancies and influenceabout 300,50 patients annually all over the world ${ }^{(1)}$. Oral squamous cell carcinoma (OSCC) is considered as $\mathbf{9 2 - 9 5 \%}$ of all oral cancers ${ }^{(2)}$. A lot ofOSCCs progress from oral potentially malignant disorders (PMDs) which supports the ideaof two-step cancer progress process ${ }^{(3)}$. Oral potentially malignant disorders (OPMDs) comprise a diversity of lesions and conditions distinguished by an increased risk for malignant transformation (MT)into oral squamous cell carcinoma (OSCC). The histopathological features of a given lesion, mostly the existence and gradeof epithelial dysplasia, are now accepted as the most functionalgauge of MT risk ${ }^{(4)}$. Severe epithelial dysplasia was present to have a malignant transformation rate of about $7-50 \%$, while mild epithelial dysplasia displays a low risk $(<5 \%)^{(5)}$.

Several natural materials have been determined as anti-cancer agentswhichavailable in vegetables and spices. Thesenatural compounds containisothiocyanates from cruciferous vegetableslike cauliflower, cabbageand similar green leafy vegetables. , catechins material from green tea, resveratrol compound from grape seeds and red wine, curcuminoids extracted from turmeric, procyanides taken from various fruits and nuts, isoflavones material from soybean, antioxidant vitamins present in various foods and finally capsaicin extracted from hot chili peppers. Presently more than 40 chemopreventive agents are undergoing clinical examination ${ }^{(6)}$.

In fact, many epidemiological and animal studies have shown that diet rich in fruits and vegetables decreased the occurrence of cancers ${ }^{(7,8)}$. Capsaicin (trans-8-methyl-N-vanillyl-6-nonenamide) is a maincomponent in hot chili pepper and has been applied for many years as food additives specially in South Asian and Latin American countries.There are many previous studies showedcapsaicin in vitro and in vivo to inhibitdifferent types of human cancers by producing reactive oxygen species (ROS) and increasing apoptosis (Programmed cell death), the suppression of mitochondrial complex activities ${ }^{(9-11)}$. The capsaicin can suppress events associated with the initiation, promotion and progression of cancer that is according to the current epidemiological data implications ${ }^{(12-15)}$. Moreover, the capsaicin can suppressthe growth of human leukemia cells, gastric, oral and hepatic carcinoma cellsin vitro and in vivo.Previous researchers studied, the anti-tumor effect of capsaicin, it can bond to transient receptor potential vanilloid 1 (TRPV1) agonist, and capsazepine, TRPV1 is also broadly distributed in tissues of the brain, bladder, kidneys, intestines, keratinocytes of epidermis, glial cells, liver, and polymorphonuclear granulocytes, mast cells, and macrophages ${ }^{(16,17)}$ a TRPV1 antagonist, on oral squamous cell carcinoma (OSCC) cell lines; the authors said that capsaicin alone reduced cell viability ${ }^{(18)}$.

Uncontrolled cell growth caused by thepiling upofgenetic and epigenetic mutations in the genes that normally play a role in the regulation of cell proliferation, thus leading to cancer.The tumorigenesis genes which include those whose products: directly regulate cell proliferation (either promoting or inhibiting), are implicated in the repair of damaged DNA and control programmed celldeath or apoptosis.The naming of these genes depends onhow they affect each process, as tumor suppressor genes (growth inhibitory), proto-oncogenes (growth promoting), or anti-apoptotic genes (inhibits apoptosis) ${ }^{(19)}$. One of anti-apoptotic protein is B-cell lymphoma/leukemia-2 (Bcl-2) that reacts with and is regulated by p53. The cell cycle and the induction of apoptosis controlled by regulatory system and $\mathrm{Bcl}-2 \mathrm{is}$ considered as a part of this system. 
The level of Bcl-2 conserve the cells from undergoing controlled cell death, and its level should be higher than the level of the death inducer, bax ${ }^{(20)}$.

The present studywas carried out to investigate immunohistochemically the effect of chemopreventive capsaicin in epithelial alteration (Epithelial dysplasia) \& carcinogenesis which induced in hamsters.

\section{MATERIAL AND METHODS}

15 goldenSyrian male hamsters five weeks old, weighting 80-120 gms were used as experimental animals and divided into three groups (G1, G2 and G3): G1 (negative control or normal group): 5 animals were left untreated. G2 (DMBA group): 5 animals right buccal pouches were painted with $0.5 \% \quad$ DMBA (7,12-dimethylbenzanthracene (Sigma-Aldrich, USA) in paraffin oil 3 times per week.G3 (capsaicin chemoprevention group): 5 animals were received capsaicin as a liquid in water(10 ppm)- week before, as well as during the application of DMBA for 8 weeks. After termination of the experiment, all animals were euthanized, and the buccal mucosa was excised and preparation to be examined histologically by $\mathrm{H}$ \& $\mathrm{E}$ and immunohistochemicallyby Bcl-2the immunostaining procedure was performed according to the manufacturer instructions.Streptavidin-biotin immunoperoxidase staining techniques was used to detect the expression of Bcl-2 in three groups.

\section{Image analysis}

Area percentage of positive staining for Bcl2 stain of tumor was measured in an area with reference to a standard measuring frame of area per 5 fields using a magnification (x 200) by light microscopy. These areas were counted and the positive index (PI) was calculated by image analyzer computer system to assess area percentage of positive cells, the image analysis was performed using a computer system (software Leica Quin 500) consisting of color video camera, color monitor, CBU of IBM personal computer connected to the microscope. This was done in the Oral and Dental Pathology Department - Faculty of Dental Medicine - Boys- Cairo - Al-Azhar University. The degree of positive staining for each antibody was evaluated by a well-established semi-quantitative scoring on a scale range from negative to strong positive staining as follow: Strong staining (more than 50\% stained), moderate staining (between 25 and 50\% stained), weak staining (between 5 and $25 \%$ stained), and negative (less than 5\% stained). Statistical presentation and analysis of the present study was conducted, using the mean, standard deviation, ANOVA and Tukey's test by SPSS V20. Significant level: Non-Significant $>0.05$ Significant $<0.05^{*}$ High Significant $<0.001^{*}$, (Table $1 \&$ Chart1).

TABLE (1) Statistical analysis between normal group (G 1), DMBA group (G 2) and capsaicin + DMBA group (G 3)

\begin{tabular}{|l|c|c|c|}
\hline & G 1 & G 2 & G 3 \\
\hline Mean & 5.240 & 57.81 & 13.37 \\
\hline Std. Deviation & 0.2434 & 0.1517 & 0.1483 \\
\hline ANOVA & \multicolumn{3}{|c|}{$<0.01 *$} \\
\hline Tukey's Post Hoc Test & \multicolumn{3}{|c|}{ (G2>G1, G3) (G3>G1) } \\
\hline
\end{tabular}

*Highly significant

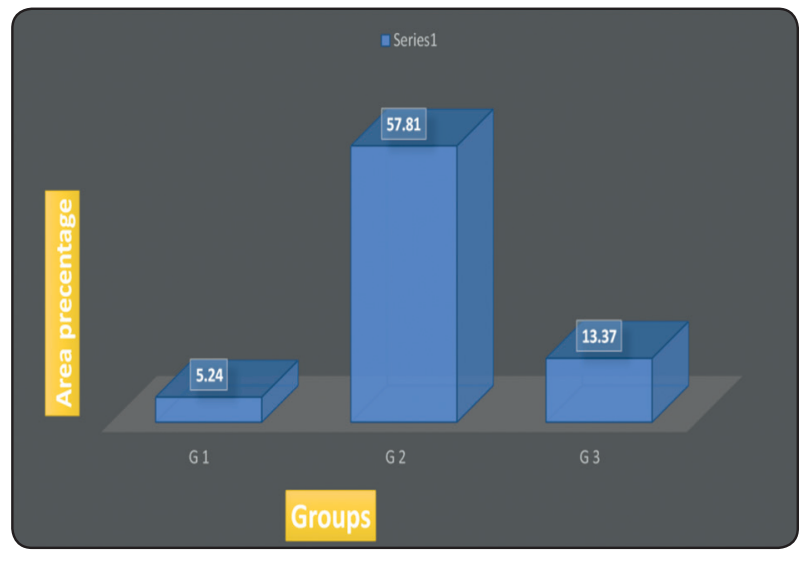

Chart (1) Mean value of area percentage of normal group (G 1), DMBA group (G 2) and capsaicin + DMBA group (G 3) 


\section{RESULTS}

Group 1 (Normal group): Clinical picture of hamster buccal pouch (Fig.1.A). Histological sections, using H\&E stain of normal epithelial tissues of buccal mucosa (Fig.1.B). Histological section, using immunohistochemical stain (Bcl-2) showed negative staining with normal epithelial tissues of buccal mucosa (Fig.1.C).

Group 2 (DMBA group):Clinical picture of hamster buccal pouch showed ulcerative lesion in mucosa (Fig.2.A). Histological sections, using H\&E stain of G2 showed $100 \%$ incidence of severe epithelial dysplasia with hyperkeratosis, acanthosis, elongation and broad bulbous rete pegs with criteria of malignancy (Fig.2.B). Histological sections, immunohistochemical stain (Bcl-2) showed strongly positive staining with severe epithelial dysplasia with acanthosis, elongation and broad of rete pegs (Fig.2.C).

Group 3 (Capsaicin + DMBA group): Clinical picture of hamster buccal pouch showed healing of ulcerative lesion in mucosa (Fig.3.A). Histological sections, using H\&E stain of G3showed reduced epithelial dysplasia incidence and severity as compared to G2, exhibited mild epithelial dysplasia(Fig.3.B). Histological sections, immunohistochemical stain (Bcl-2) showed mild (weak) positive staining with mild epithelial dysplasia(Fig.3.C).

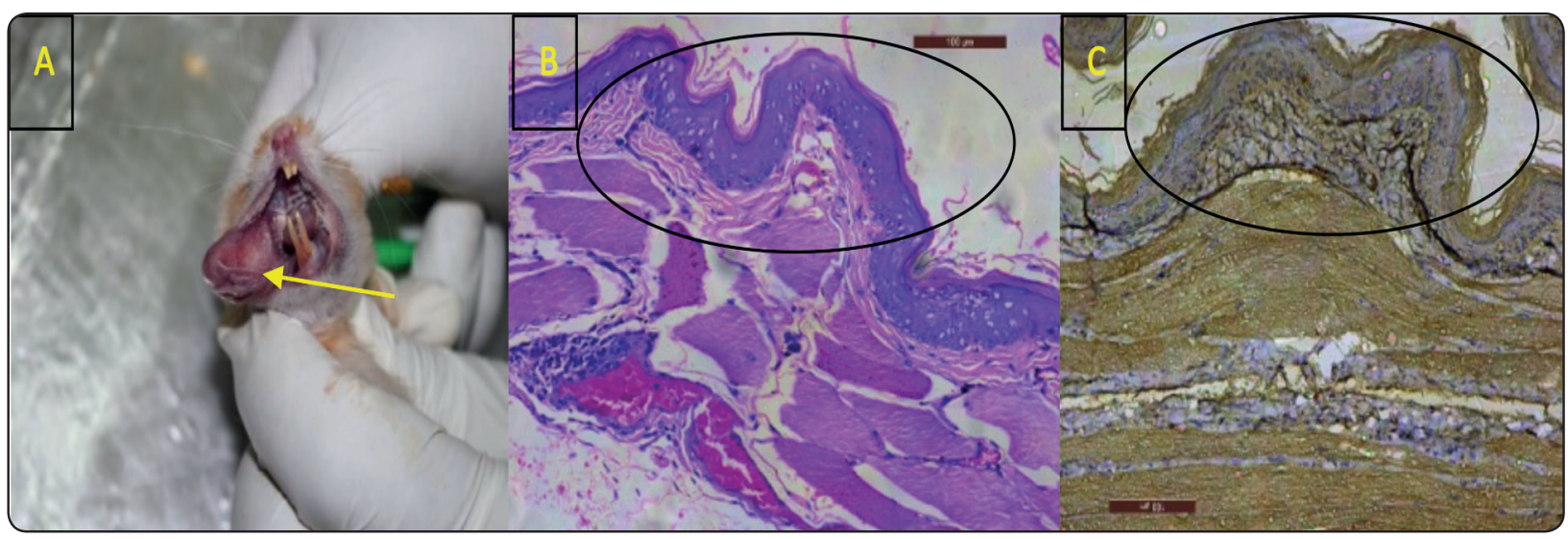

Fig. 1 A) Normal clinical features of hamster buccal pouch. B) Histological section,using H\&E stain showed normal epithelial tissues of buccal mucosa (X 100).C) Histological section, using immunohistochemical stain (Bcl-2) showed negative staining with normal epithelial tissues of buccal mucosa (X 200).
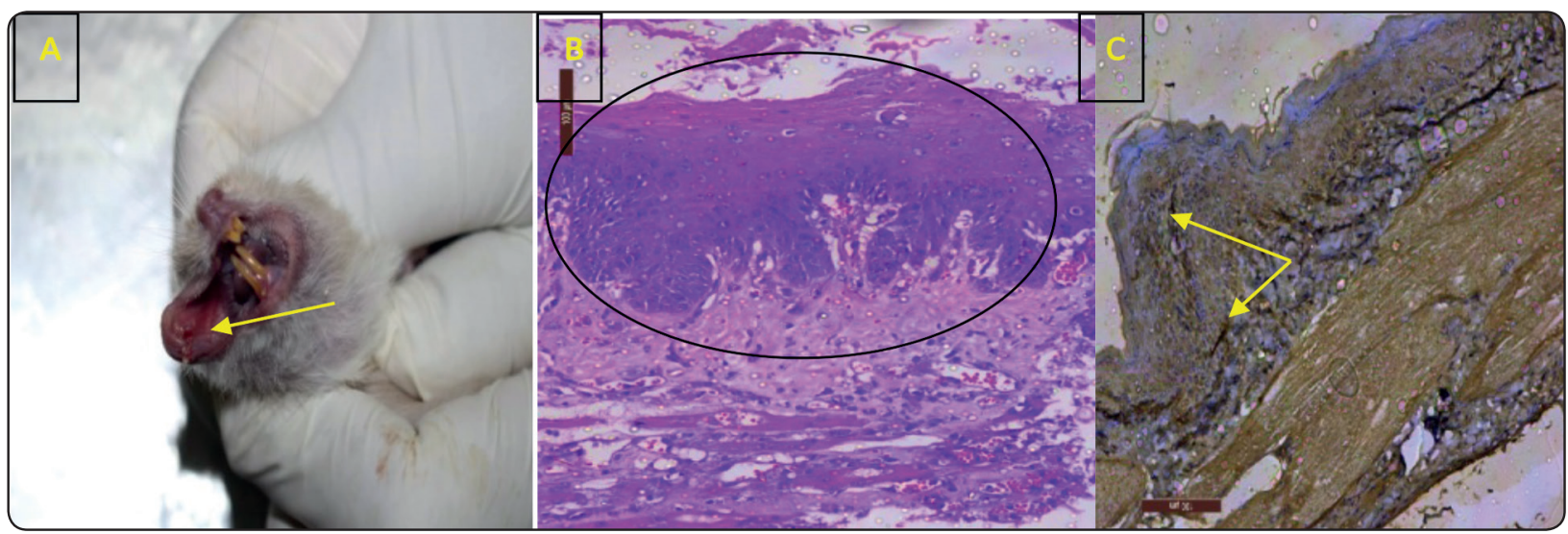

Fig. 2 A) Clinical picture of hamster buccal pouch showed ulcerative lesion in mucosa. B) Histological section,using H\&E stain showed severe epithelial dysplasia with hyperkeratosis, acanthosis, elongation and broad bulbous rete pegs with criteria of malignancy (X 100).C) Histological section, using immunohistochemical stain (Bcl-2) showed strongly positive staining with severe epithelial dysplasia with acanthosis, elongation and broad of rete pegs (X 200). 


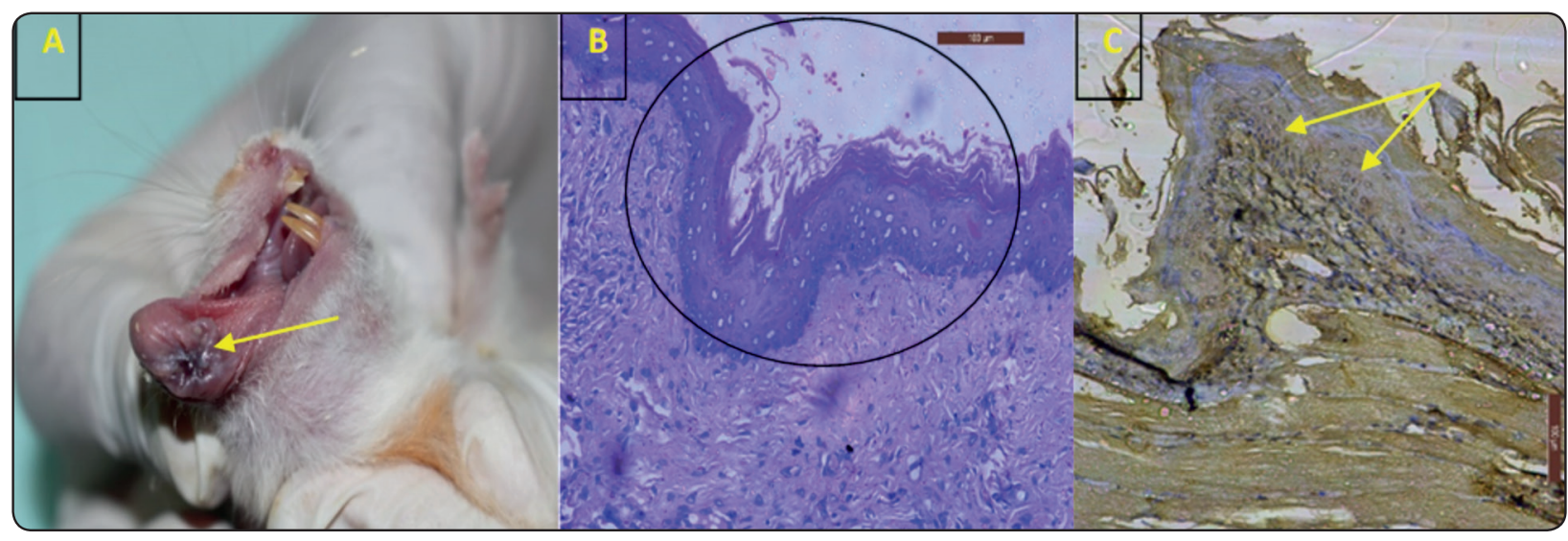

Fig. 3 A) Clinical picture of hamster buccal pouch healing of ulcerative lesion in mucosa. B) Histological sections, using H\&E stain of G3showed reduced epithelial dysplasia incidence and severity as compared to G2, exhibited mild epithelial dysplasia (X 100).C) Histological section, using immunohistochemical stain (Bcl-2) showed mild (weak) positive staining with mild epithelial dysplasia (X 200).

\section{DISCUSSION}

Abnormal, atypical proliferation encountered fundamentally in the epithelium means oral epithelial dysplasia. Epithelial dysplasia, dyskeratosis and epithelial atypia were the terms usedsynonymous$1 y^{(21)}$. The sense of a disordered development is generally enrolled in as the term "dysplasia". In a stratified squamous epithelium, the normal stratification and maturity affected byarchitectural disorder ${ }^{(22)}$. The cancer progression is believed to be associated with epithelial dysplasia in the oral mucosa. The sever epithelial dysplasia may be progressed to malignancy in an individual lesion. However, nondysplastic lesions may also show malignant growth ${ }^{(23,24)}$. Epithelial dysplasia mostly appeared in clinical features as a red,white or mixed lesion. There is an association between the risk of malignant transformation and the histological severity of the lesion so the histological grading play an important role in the transformation ${ }^{(25)}$.

In the present study, all over the world capsaicin is considered as one of the most commonly used spices so we used it to investigate its ability as anti-carcinogenic agent and tumor suppression. According to Tukey's test there were highly differences between the normal group (G 1), capsaicin + DMBA group (G 3) and DMBA group (G 2) in oral mucosa which appeared as area percentage of Bcl-2 $(\mathrm{G} 2>\mathrm{G} 1, \mathrm{G} 3)$ but also there were difference between $\mathrm{G} 1 \& \mathrm{G} 3$ as a recorded by the same test $(\mathrm{G} 3>\mathrm{G} 1)$ so there were highly significant( $\mathrm{p}$ $\leq 0.01$ ) among the three different groups, this results of immunohistochemical expression Bcl-2 confirms that the effect of capsaicin in induced oral epithelial dysplasia(capsaicin + DMBA group (G 3) has a slower proliferative rate of cell proliferation that related to tumor aggressiveness, play an important role in regulation of cell cycle and better prognosis compared to that of group 2(DMBAgroup (G2). This result similar to the observations of Anandakumar et $\mathrm{al}^{(26)}$, described that capsaicin is effective in regulating the components of extracellular matrix and the proteases, which in turn paves the way for its anti-cancer action against experimentally induced lung cancer. Also, the present study agrees with previous study of Le et al ${ }^{(27)}$. which studied the effect of capsaicin on humanpharyngeal squamous cell carcinoma(SCC) cells andfoundthat capsaicin inhibits growth and proliferation in a time and dose dependent manner and induces apoptosis via mitochondrial pathways. They also analyzed the expression of the anti-apoptotic Bcl-2 gene found a reduction of Bcl-2 gene in the humanpharyngeal squamous cell carcinoma. 
In the present study,DMBA group (G 2) showed statistically significant highest mean $\mathrm{Bcl}-2$ area percentage (57.81) than capsaicin + DMBA group (G 3) 13.37 with $P$ value less than $<0.01$ so the epithelial dysplasia was reduced in group 3 compare to group 2 due to the effect of capsaicin and when we compare the area percentage of group 3 (13.37) \& control group G1 (5.240)(G3>G1) so the histological features of group 3 nearly resemble to group 1 . These findings may be supported by those of Gonzales et $\mathrm{al}^{(16)}$. who reported that the antitumor effect of capsaicin, a TRPV1 agonist, and capsazepine, a TRPV1 antagonist, on oral squamous cell carcinoma (OSCC) cell lines; the authors found that capsaicin alone reduced cell viability.

\section{CONCLUSION}

Capsaicin play an important role in reduction and prevention of transformation of epithelial dysplasia into malignant tumor especially oral squamous cell carcinoma. The liquid form of capsaicin may be considered as a new line of treatment in oral dysplasia or even in oral squamous cell carcinoma and the Bcl-2 expression evidenced this reduction of abnormality in epithelial dysplasia.

\section{REFERENCES}

1- Torre LA, Bray F, Siegel RL, Ferlay J, Lortet-Tieulent J, Jemal A. Global cancer statistics, 2012. CA: a cancer journal for clinicians. 2015;65:87-108.

2- George A, Sreenivasan B, Sunil S, Varghese SS, Thomas J, Gopakumar D, Mani V. Potentially malignant disorders of oral cavity. Oral \& Maxillofacial Pathology Journal. 2011;2: 95-100.

3- Reibel J. Prognosis of Oral Pre-malignant Lesions: Significance of Clinical, Histopathological, and Molecular Biological Characteristics. Critical Reviews in Oral Biology \& Medicine. 2003 January 1, 2003;14:47-62.

4- Scully C, Sudbø J, Speight PM. Progress in determining the malignant potential of oral lesions. Journal of Oral Pathology \& Medicine. 2003;32:251-56.

5- Bouquot JE, Speight PM, Farthing PM. Epithelial dysplasia of the oral mucosa-Diagnostic problems and prognostic features. Current Diagnostic Pathology. 2006;12:11-21.
6- Keum YS, Jeong WS, Kong AN Chemoprevention by isothiocyanates and their underlying molecular signaling mechanisms. Mutat Res. 2004; 555:191-202.

7- Benetou V, Orfanos P, Lagiou P, Trichopoulos D, Boffetta $\mathrm{P}$, Trichopoulou A. Vegetables and fruits in relation to cancer risk: evidence from the greek EPIC cohort study. Cancer Epidemiol Biomarkers Prev. 2008;17(2):387-92.

8- Freedman ND, Park Y, Subar AF, Hollenbeck AR, Leitzmann MF, Schatzkin A, et al. Fruit and vegetable intake and head and neck cancer risk in a large United States prospective cohort study. Int J Cancer. 2008;122(10):2330-36.

9- Zhang R, Humphreys I, Sahu RP, Shi Y, Srivastava SK. In vitro and in vivo induction of apoptosis by capsaicin in pancreatic cancer cells is mediated through ROS generation and mitochondrial death pathway. Apoptosis. 2008; 13:1465-78.

10- Lee YS, Kang YS, Lee JS, Nicolova S, Kim JA. Involvement of NADPH oxidase-mediated generation of reactive oxygen species in the apoptotic cell death by capsaicin in HepG2 human hepatoma cells. Free Radic Res. 2004b; 38:405-12.

11- Pramanik KC, Boreddy SR, Srivastava SK. Role of mitochondrial electron transport chain complexes in capsaicin mediated oxidative stress leading to apoptosis in pancreatic cancer cells. PLoS One. 2011;6: e20151: 1-16.

12- Modly CE, Das M, Don PS, Marcelo CL, Mukhtar H, Bickers DR. Capsaicin as an in vitro inhibitor of benzo(a) pyrene metabolism and its DNA binding in human and murine keratinocytes. Drug MetabDispos Biol Fate Chem. 1986; 14:413-16.

13- Surh YJ, Lee SS. Capsaicin, a double-edged sword: toxicity, metabolism, and chemopreventive potential. Life Sci. 1995; 56:1845-55.

14- Teel RW. Effects of capsaicin on rat liver S9-mediated metabolism and DNA binding of aflatoxin. Nutr Cancer. $1991 ; 15: 27-32$.

15- Yoshitani SI, Tanaka T, Kohno H, Takashima S. Chemoprevention of azoxymethane- induced rat colon carcinogenesis by dietary capsaicin and rotenone. Int $\mathrm{J}$ Oncol. 2001; 19:929-39.

16- Cortright, DN.; Szallasi, A. Biochemical pharmacology of the vanilloid receptor TRPV1. Eur. J. Biochem. 2004; 271, 1814-19.

17- Tominaga, M.; Tominaga, T. Structure and function of TRPV1. Pflugers Arch. 2005; 451, 143-50. 
18- Gonzales CB, Kirma NB, Jorge J, Chen R, Henry MA, Luo S,et al. Vanilloids induce oral cancer apoptosis independent of TRPV1. Oral Oncol. 2014; 50, 437-47. [CrossRef] [PubMed].

19- Rahmani A, Alzohairy M, Babiker AY, Rizvi MA, Elkarimahmad HG. Clinicopathological significance of PTEN and bcl2 expressions in oral squamous cell carcinoma. Int J Clin Exp Pathol. 2012;5: 965-71.

20- Korsmeyer SI. Regulators of cell death.Trends Genet. 1995;11:101-05.

21- Sharma N, Hosmani JV, Tiwari V. Epithelial dysplasia: different grading system and its applications. J Int Oral Health. 2010;1-16.

22- Kujan O, Richard JO, Khattab A, Stephen A. Evaluation of a new binary system of grading oral epithelial dysplasia for prediction of malignant transformation. Oral Oncol. 2006;42(10):987-93.

23- Gale N, Plich BZ, Sidransky D, Westra W, Califano J. Tumours of the hypopharynx, larynx and trachea (Epithelial precursor lesions). In: Barnes L, Eveson JW, Reichart
$\mathrm{P}$ and Sidransky D, eds. World Health Organization Classification of Tumours Pathology \& Genetics Head and Neck Tumours International Agency for Research on Cancer (IARC) (Lyon: IARC Press), 2005; pp. 140-43.

24- Warnakulasuriya S, Reibel J, Bouquot J, Dabelsteen E. Oral epithelial dysplasia classification systems: predictive value, utility, weaknesses and scope for improvement. J Oral Pathol Med. 2008; 37: 127-33.

25- Lee JJ, Hong WK, Hittelman WN, Mao L, Lotan R, Shin DM, et al. Predicting cancer development in oral leukoplakia: ten years of translational research. Clin. Cancer Res. 2000;6:1702-10.

26- Anandakumar P, Kamaraj S, Jagan S, Ramakrishnan G, Asokkumar S, Naveenkumar C, et al.The anticancer role of capsaicin in experimentally induced lung carcinogenesis. Journal of Pharmacopuncture 2015;18(2):019-25.

27- Le, T.D.; Jin, D.C.; Rho, S.R.; Kim, M.S.; Yu, R.; Yoo, H. Capsaicin-induced apoptosis of $\mathrm{FaDu}$ human pharyngeal squamous carcinoma cells. Yonsei Med. J. 2012; 53, 834 41. [CrossRef] [PubMed]. 